\title{
Reflection, Transmission, and Absorption of Vortex Beams Propagation in an Inhomogeneous Magnetized Plasma Slab
}

\author{
Haiying Li, Farideh Honary, Zhensen Wu, Senior Member, IEEE, Qingchao Shang, and Lu Bai
}

\begin{abstract}
Based on the angular spectrum expansion and the $4 \times 4$ transfer matrix method, an investigation into the reflection, transmission, and absorption of vortex beams in an inhomogeneous magnetized plasma slab is presented. The reflected and transmitted electric fields are expressed by the inverse Fourier transform of the product of the reflected and transmitted coefficients and the angular spectrum amplitude of the incident beam. The intensity profiles, as well as the distortion of OAM states in both the reflected and transmitted beams are simulated and discussed. Through this investigation it could be concluded that both the incident angle and the plasma parameters have significant impact on the magnitudes of reflected and transmitted intensities, and the distortion of OAM states. The effects of the magnetic field and the incident angle on the reflectance, transmittance, and absorptance of the power have also been reported.
\end{abstract}

Index Terms-Electromagnetic propagation in plasma media, Electromagnetic reflection, Electromagnetic refraction, Fourier transforms, Nonhomogeneous media, Numerical analysis.

\section{INTRODUCTION}

INCE THE orbital angular momentum (OAM) of the Laguerre Gaussian(LG) beam was discovered in 1992 [1], the characteristics of vortex light beams [2]-[6] have become an important area of research and thus attracting the researchers to deeply explore further. In 2007, Thidé firstly introduced OAM into the low-frequency radio domain [7]. Since then, the methods of generation of vortex beams in microwave and radio bands have been reported extensively in the literature [8]-[11]. Besides, the characteristics and applications of vortex microwave and radio beams, particularly in wireless communication [12]-[15] and radar target imaging

This work was supported by National Natural Science Foundation of China under grants 61571355, 61475123, and 61701382, Natural Science Basic

Research Plan in Shaanxi Province of China under grant 2016JQ4015, the 111 Project under grants B17035, the Fundamental Research Funds for the Central Universities, and the overseas training program for young backbones teachers sponsored by China Scholarship Council (CSC) and Xidian University.

Haiying Li is with the School of Physics and Optoelectronic Engineering, Xidian University, Xi'an, 710071, China; Collaborative Innovation Center of Information Sensing and Understanding at Xidian University, Xi'an, 710071, China. (e-mail: lihy@ xidian.edu.cn).

Farideh Honary is with the Department of physics, Lancaster University, Lancaster, LA1 4YB, U.K..

Zhensen Wu, Qingchao Shang, and Lu Bai are with the School of Physics and Optoelectronic Engineering, Xidian University, Xi'an, 710071, China.
[16], [17], have been the focus of attention. This is due to the fact that vortex beams can potentially increase the system capacity and spectral efficiency of wireless communication [12], and also have the potential to acquire the cross-range profile of the target [16].

Plasma is the fourth state of matter and is ubiquitous in the universe, such as in the sun and other stars, in the interplanetary interstellar, medium as well as in the Earth's ionosphere. A number of investigations into the interaction of space plasma with vortex radio beams have been presented through the ionospheric HF-heating experiments [18]-[20], in which the effects of vortex beams on the features of simulated electromagnetic emissions are analyzed. The reflection and attenuation of electromagnetic waves caused by ionospheric plasma can have a great influence on the radio communication, satellite navigation, and positioning. The plasma sheath on the surface of hypersonic vehicles may cut off the communication with anything outside the vehicle. Therefore, the effects of plasma on the propagation of electromagnetic waves need to be considered. Also, a great deal of attention [21]-[30] on the interaction between electromagnetic waves and plasma has been conducted in the past few decades. Using scattering matrix method (SMM), Tang et al. [22] analyzed the broadband absorption characteristics of magnetized non-uniform plasma. Jazi et al. [24] provided an analytical method to study the reflection and absorption of electromagnetic waves in an inhomogeneous dissipative magnetized plasma slab, and also discussed the effects of an external magnetic field, the frequency of an incident wave, as well as the electron thermal velocity. The finite-difference time-domain (FDTD) method was also successfully employed to simulate the electromagnetic waves propagating in magnetized plasma [25]-[28]. The $4 \times 4$ transfer matrix method which was established by Berreman [31] and Yeh [32], is indeed a very efficient approach to analyze the propagation of electromagnetic waves in anisotropic media. However, most of the existing work mainly focuses on the propagation of plane waves in plasmas, not including vortex beams. Considering the promising prospects in the practical applications of vortex beams in the radio communications, plasma diagnostics, and ionospheric heating, the investigations into the propagation of vortex beams in plasmas is the next step forward which needs to be developed. In this work, the reflection, transmission, and absorption of vortex beams in an inhomogeneous magnetic plasma slab based on the theories of angular spectrum expansion and the $4 \times 4$ transfer matrix method have been considered. Taking LG modes as an example, the 
reflected and transmitted electric fields, as well as the reflectance and transmittance of the power are expressed by the inverse Fourier transform of the angular spectrum. As the direction of an external magnetic field is parallel to the $z$ axis, the reflected and transmitted coefficients are obtained through the $4 \times 4$ transfer matrix method. By using the two-dimensional (2D) midpoint rule of integrals, the intensity profiles and distortion of OAM states of the reflected and transmitted beams are simulated in detail. The effects of the magnetic field and the incident angle on reflectance, transmittance, and absorptance of the power are also discussed.

This paper is organized into four sections. Section I gives the introduction. The theoretical formulae of the reflected and transmitted electric fields, as well as the reflectance, transmittance, and absorptance of the power are presented in Section II. The numerical results of the reflected and transmitted intensities, the distribution of OAM states, as well as the reflectance, transmittance, and absorptance of the power have been shown along with the corresponding discussion in Section III. Section IV provides the summary and conclusion of this work.

\section{METHODOLOGY}

\section{A. Physical Model}

Assuming an LG vortex beam which is incident on the plasma slab-vacuum interface, the physical model as shown in Fig. 1 represents the positions of the global coordinate system $(x, y, z)$, the incident coordinate system $\left(x_{i}, y_{i}, z_{i}\right)$, the reflected coordinate system $\left(x_{r}, y_{r}, z_{r}\right)$, and the transmitted coordinate system $\left(x_{t}, y_{t}, z_{t}\right)$. The $x_{\alpha}-y_{\alpha}$ planes in the local coordinate systems $\left(x_{\alpha}, y_{\alpha}, z_{\alpha}\right) \quad \alpha=i, r$ or $t$ define the transverse planes of the incident, the reflected, and the transmitted beams, respectively, where the $z_{\alpha}$ axis is coincident with the central axis of the corresponding beam. The inhomogeneous magnetized plasma slab is stratified into $N$ layers, and the electron number density is uniform in each of the layers. $h_{j}$ is the thickness of the $j^{\text {th }}$ layer. The incident angle is denoted by $\theta_{i}$. Whereas, $\theta_{r}$ and $\theta_{t}$ represent the reflected and the transmitted angles, respectively.

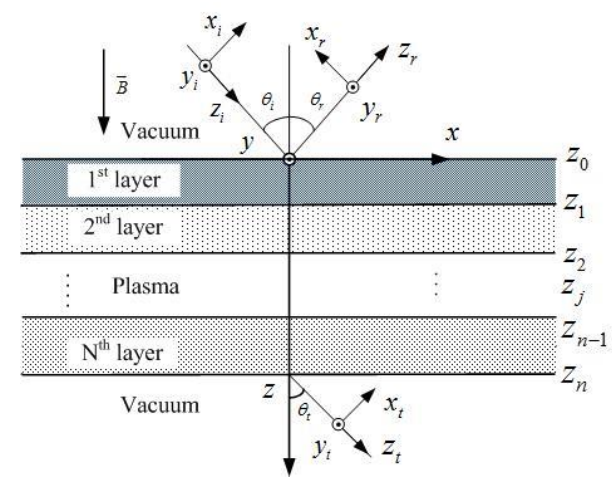

Fig.1 The physical model of a vortex beam incident on an inhomogeneous magnetized plasma slab
In the focal plane, the complex electric field amplitude of the linearly polarized LG mode in the incident coordinate system is written as follows

$$
E_{i}\left(r_{i}, \phi_{i}, z_{i}=0\right)=E_{0}\left[\frac{\sqrt{2} r_{i}}{w_{0}}\right]^{|l|} \exp \left[\frac{-r_{i}^{2}}{w_{0}^{2}}\right] L_{p}^{|l|}\left(\frac{2 r_{i}^{2}}{w_{0}^{2}}\right) \exp \left(i l \phi_{i}\right),
$$

where the subscript $i$ represents the incident wave in the incident coordinate system, $E_{0}$ is the amplitude constant, $w_{0}$ is the beam waist radius at $z_{i}=0$, and $L_{p}^{|l|}(\cdot)$ is the associated Laguerre polynomial. $p$ and $l$ represent the radial index and the topological charge of the LG mode, respectively. It should be noticed that (1) is given in a cylindrical coordinate system which is linked with Cartesian coordinate system by $r_{i}=\sqrt{x_{i}^{2}+y_{i}^{2}}$ and $\phi_{i}=\operatorname{atan}\left(y_{i} / x_{i}\right)$. The time dependence of $\exp (-i \omega t)$ is omitted.

As the direction of external magnetic field is parallel to the $z$ -axis, the dielectric tensor of magnetized plasma can be written as follows:

$$
\varepsilon=\left[\begin{array}{ccc}
\varepsilon_{1} & i \varepsilon_{2} & 0 \\
-i \varepsilon_{2} & \varepsilon_{1} & 0 \\
0 & 0 & \varepsilon_{3}
\end{array}\right]
$$

The three elements in (2) are represented as follows:

$$
\begin{aligned}
& \varepsilon_{1}=\varepsilon_{0}\left(1+\frac{\omega_{p}^{2}(\omega+i v)}{\omega\left[\omega_{c}^{2}-(\omega+i v)^{2}\right]}\right), \\
& \varepsilon_{2}=\varepsilon_{0}\left(\frac{\omega_{p}^{2} \omega_{c} / \omega}{\omega_{c}^{2}-(\omega+i v)^{2}}\right), \\
& \varepsilon_{3}=\varepsilon_{0}\left(1-\frac{\omega_{p}^{2}}{\omega(\omega+i v)}\right),
\end{aligned}
$$

where $\varepsilon_{0}$ is the dielectric constant of vacuum, $\omega_{c}=e B_{0} / m$ is the electron cyclotron frequency, $v$ is the electron collision frequency, $\omega_{p}$ is the angular plasma frequency, and $\omega$ is the angular frequency of the incident beam. The charge and mass of an electron are $e=1.6 \times 10^{-19} \mathrm{C}$ and $m=9.11 \times 10^{-31} \mathrm{~kg}$ respectively. $B_{0}$ is the magnetic flux intensity.

\section{B. Reflection and transmission of vortex beams in an inhomogeneous magnetized plasma slab}

Based on the angular spectrum method, the complex amplitude function of the linearly polarized incident LG beam is given by the following (6)[33],

$$
\begin{gathered}
\bar{E}_{i}\left(k_{i}, \varphi_{i}\right)=E_{0} i^{l} \exp \left(i l \varphi_{i}\right)\left(\frac{w_{0}}{\sqrt{2}}\right)^{2}(-1)^{p+1} . \\
\left(\frac{k_{i}}{\sqrt{2} k_{0} f}\right)^{l} \cdot \exp \left(-\frac{k_{i}^{2}}{4 k_{0}^{2} f^{2}}\right) L_{p}^{|l|}\left(\frac{k_{i}^{2}}{2 k_{0}^{2} f^{2}}\right),
\end{gathered}
$$

where $k_{0}$ is the wavenumber in vacuum, and $f=1 / k_{0} w_{0}$ is the natural expansion factor[34]. The relationship between the 
wave number components $\left(k_{x}, k_{y}, k_{z}\right)$ and $\left(k_{x \alpha}, k_{y \alpha}, k_{z \alpha}\right)$ $\alpha=i, r$ or $t$ is given by $k_{x}=k_{x \alpha} \cos \theta_{\alpha}+\sqrt{k_{0}{ }^{2}-k_{x \alpha}{ }^{2}-k_{y \alpha}{ }^{2}} \sin \theta_{\alpha}$, $k_{y}=k_{y \alpha}$, and $k_{z}=-k_{x \alpha} \sin \theta_{\alpha}+\sqrt{k_{0}^{2}-k_{x \alpha}{ }^{2}-k_{y \alpha}{ }^{2}} \cos \theta_{\alpha}$.

The reflected and transmitted electric fields in the local coordinate systems can be expressed by the inverse Fourier transform as shown in the following (7) and (8):

$$
\begin{aligned}
E_{r}\left(r_{r}, \phi_{r}\right)= & \frac{1}{2 \pi} \int_{0}^{\infty} \int_{0}^{2 \pi} r \bar{E}_{i}\left(k_{i}, \varphi_{i}\right) \exp \left[i k_{r} r_{r} \cos \left(\phi_{r}-\varphi_{r}\right)\right] . \\
& \exp \left[i k_{z r}\left(z_{1}+z_{2}\right)\right] k_{r} d k_{r} d \varphi_{r} \\
E_{t}\left(r_{t}, \phi_{t}\right)= & \frac{1}{2 \pi} \int_{0}^{\infty} \int_{0}^{2 \pi} t \bar{E}_{i}\left(k_{i}, \varphi_{i}\right) \exp \left[i k_{t} r_{t} \cos \left(\phi_{t}-\varphi_{t}\right)\right] . \\
& \exp \left[i\left(k_{z i} z_{1}+k_{z t} z_{3}\right)\right] k_{t} d k_{t} d \varphi_{t}
\end{aligned}
$$

where $r$ and $t$ in the integral functions are the total amplitudes of the reflected and transmitted coefficients of the inhomogeneous plasma slab, respectively. The subscripts $r$ and $t$ represent the reflected and the transmitted coordinate systems, respectively. $z_{1}, z_{2}$, and $z_{3}$ are the distances between the center of the global coordinate system and the center of the incident, the reflected, and the transmitted coordinate systems, respectively. $k_{z \alpha}=\sqrt{k_{\alpha}{ }^{2}-k_{x \alpha}{ }^{2}-k_{y \alpha}{ }^{2}}(\alpha=i, r$ or $t)$ are the longitudinal components of wave vector in local coordinate systems $\left(x_{\alpha}, y_{\alpha}, z_{\alpha}\right)$.

According to the Maxwell's equations, we can obtain the intensity i.e. $I \infty|E|^{2}$. The formulae for the reflectance and transmittance of the power can be written as follows:

$$
\begin{gathered}
R=\frac{\int_{0}^{\infty} \int_{0}^{2 \pi}\left|E_{r}\left(r_{r}, \phi_{r}, z_{r}\right)\right|^{2} r_{r} d r_{r} d \phi_{r}}{\int_{0}^{\infty} \int_{0}^{2 \pi}\left|E_{i}\left(r_{i}, \phi_{i}, z_{i}\right)\right|^{2} r_{i} d r_{i} d \phi_{i}}, \\
T=\frac{\int_{0}^{\infty} \int_{0}^{2 \pi}\left|E_{t}\left(r_{t}, \phi_{t}, z_{t}\right)\right|^{2} r_{t} d r_{t} d \phi_{t}}{\int_{0}^{\infty} \int_{0}^{2 \pi}\left|E_{i}\left(r_{i}, \phi_{i}, z_{i}\right)\right|^{2} r_{i} d r_{i} d \phi_{i}}
\end{gathered}
$$

Thus, the absorptance of the power is $A=1-R-T$.

\section{Reflected and transmitted coefficients of the inhomogeneous magnetized plasma slab by $4 \times 4$ transfer matrix method}

Supposing that the input and output regions are isotropic media, using the $4 \times 4$ transfer matrix method, the reflected and transmitted coefficients [31] can be derived from the transfer matrix as follows,

$$
\bar{T}=\Psi_{t}^{-1}\left[\prod_{j=1}^{N} P_{j}\left(h_{j}\right)\right] \Psi_{i},
$$

where $P_{j}\left(h_{j}\right)$ describes the propagation of plane wave in the $j^{\text {th }}$ layer, which could be expressed in the following (12) [35],

$$
P_{j}\left(h_{j}\right)=\beta_{0} \bar{I}-\beta_{1} \bar{\Delta}_{j}+\beta_{2} \bar{\Delta}_{j}{ }^{2}-\beta_{3} \bar{\Delta}_{j}{ }^{3} .
$$

where $\bar{I}$ is a $4 \times 4$ identity matrix. The coefficients $\beta_{j}$ ( $j=0,1,2,3)$ are determined by the expanded equations of $\exp \left(i k_{0} \tilde{k}_{z m, j} h_{j}\right) . \tilde{k}_{z m, j}=k_{z m, j} / k_{0} \quad(m=1,2,3,4)$ represent the normalized longitudinal components of wave vector as follows:

$$
\tilde{k}_{z_{2}, j}= \pm\left[a_{1}-\left(a_{1}^{2}-a_{2}\right)^{1 / 2}\right]^{1 / 2}, \tilde{k}_{z_{4}^{3}, j}= \pm\left[a_{1}+\left(a_{1}^{2}-a_{2}\right)^{1 / 2}\right]^{1 / 2},
$$

where

$$
\begin{aligned}
& a_{1}=-\frac{\left(\tilde{k}_{x, j}^{2}+\tilde{k}_{y, j}^{2}\right)\left(\tilde{\varepsilon}_{1, j}+\tilde{\varepsilon}_{3, j}\right)}{2 \tilde{\varepsilon}_{3, i}}+\tilde{\varepsilon}_{1, j} \\
& a_{2}=\frac{\tilde{\varepsilon}_{1, j}\left(\tilde{k}_{x, j}^{4}+\tilde{k}_{y, j}^{4}\right)+2 \tilde{k}_{x, j}^{2} \tilde{k}_{y, j}^{2} \tilde{\varepsilon}_{1, j}-\left(\tilde{k}_{x, j}^{2}+\tilde{k}_{y, j}^{2}\right)\left(\tilde{\varepsilon}_{1, j}{ }^{2}-\tilde{\varepsilon}_{2, j}{ }^{2}\right)}{\tilde{\varepsilon}_{3, j}}- \\
& \tilde{k}_{x, j}^{2} \tilde{\varepsilon}_{1, j}-\tilde{k}_{y, j}^{2} \tilde{\varepsilon}_{1, j}+\tilde{\varepsilon}_{1, j}{ }^{2}-\tilde{\varepsilon}_{2, j}{ }^{2}
\end{aligned}
$$

The matrix $\bar{\Delta}_{j}$ in (12) is written as follows,

$$
\left[\begin{array}{cccc}
0 & 1-\frac{\tilde{k}_{x, j}^{2}}{\tilde{\varepsilon}_{3}} & 0 & \frac{\tilde{k}_{x, j} \tilde{k}_{y, j}}{\tilde{\varepsilon}_{3}} \\
\tilde{\varepsilon}_{1, j}-\tilde{k}_{y, j}^{2} & 0 & j \tilde{\varepsilon}_{2, j}+\tilde{k}_{x, j} \tilde{k}_{y, j} & 0 \\
0 & -\frac{\tilde{k}_{x, j} \tilde{k}_{y, j}}{\tilde{\varepsilon}_{3, j}} & 0 & \frac{\tilde{k}_{y, j}^{2}}{\tilde{\varepsilon}_{3, j}}-1 \\
j \tilde{\varepsilon}_{2}, j-\tilde{k}_{x, j} \tilde{k}_{y, j} & 0 & \tilde{k}_{x, j}^{2}-\tilde{\varepsilon}_{1, j} & 0
\end{array}\right],
$$

where $\quad \tilde{k}_{x, j}=k_{x, j} / k_{0} \quad, \quad \tilde{k}_{y, j}=k_{y, j} / k_{0}, \quad \tilde{\varepsilon}_{1, j}=\varepsilon_{1, j} / \varepsilon_{0}$, $\tilde{\varepsilon}_{2, j}=\varepsilon_{2, j} / \varepsilon_{0}, \tilde{\varepsilon}_{3, j}=\varepsilon_{3, j} / \varepsilon_{0} \cdot k_{x, j}$ and $k_{y, j}$ are the transverse components of wave vector in the global coordinate system $(x, y, z) . \Psi_{i}$ and $\Psi_{t}$ denote the relationship between the incident and transmitted tangential field components in the input and output regions [36].

Substituting the total reflected and transmitted coefficients into (7) and (8), the magnitudes of the reflected and transmitted fields can be integrated by the $2 \mathrm{D}$ midpoint rule.

\section{NUMERICAL RESULTS AND DISCUSSION}

\section{A. Intensity profiles of the reflected and transmitted beams}

In order to verify the accuracy of the $4 \times 4$ transfer matrix method followed in this investigation, for the plane wave incident normally upon a magnetized plasma slab, the reflectance of the power is plotted in Fig. 2 and has been compared with the results of SMM [37].The parameters of the slab are $h=1.5 \mathrm{~cm}, v=20 \mathrm{GHz}, \omega_{p}=2 \pi \times 28.7 \times 10^{9} \mathrm{rad} / \mathrm{s}$ and $\omega_{c}=8.8 \times 10^{10} \mathrm{rad} / \mathrm{s}$. It could be clearly observed from Fig. 2 that the results computed with the $4 \times 4$ transfer matrix method are in good agreement with SMM.

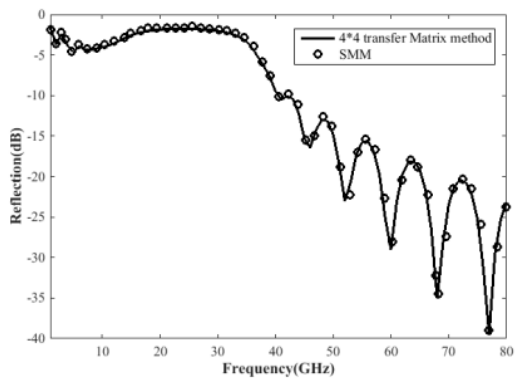

Fig.2. Reflection of the power from a magnetized plasma slab

Assuming that both the upper and the bottom of the plasma slabs are in vacuum, Figs. 3 and 4 show the intensity profiles of the reflected and transmitted beams. Each figure demonstrates 
8 panels, where both the TE and TM polarized incident beams are considered simultaneously. Also, the characteristics of cross-polarization components are presented individually. As the incident beam is TE polarized, Figs. 3 (a), (b), (e), and (f) are the intensity profiles of the TE polarized reflected beam, the TM polarized reflected beam, the TE polarized transmitted beam, and the TM polarized transmitted beam, respectively. Whereas, Figs. 3 (c), (d), (g), and (h) are the corresponding results for the TM polarized incident beam.

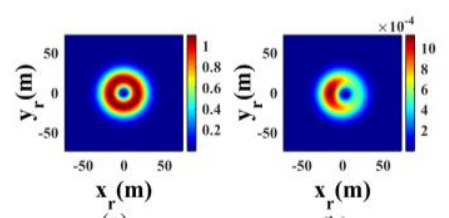

(a)

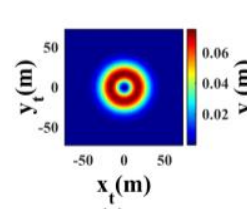

(e)

Fig. 3 Intensity profiles of the reflected and transmitted beams from a homogeneous plasma slab, $B_{0}=0.003 T, p=0, l=1$. (a)TE-TE reflected beam; (b)TE-TM reflected beam; (c)TM-TM reflected beam; (d)TM-TE reflected beam; (e)TE-TE transmitted beam; (f) TE-TM transmitted beam; (g) TM-TM transmitted beam; (h) TM-TE transmitted beam

Fig. 3 shows the intensity profile results from a homogeneous plasma slab. The incident wave frequency is $2.5 \mathrm{GHz}$. The beam waist radius is $200 \lambda$. The incident angle is at $30^{\circ}$. In the slab, the electron number density is $n_{e}=1.7 \times 10^{16} / \mathrm{m}^{3}$, the thickness of slab is $10 \lambda$, and the collision frequency is set to $10 \mathrm{GHz}$. The distances $z_{1}, z_{2}$, and $z_{3}$ are set to $0.1 \mathrm{~m}$.

Fig. 3 reveals that when the linearly polarized incident beam is in TE/TM modes, the reflected and transmitted beams have $\mathrm{TM} / \mathrm{TE}$ polarized elements (cross-polarization components), which are smaller than the corresponding TE/TM polarized elements (principal-polarization components). It is also noted from Fig.3 (e), (f), (g), and (h) that for the TE/TM polarized incident LG beam, the intensity contours of the transmitted beams have a circular shape, similar to that of an incident beam. However, the intensity contours of the reflected beams are distorted, especially for the cross-polarization components as shown in Fig.3 (b), and Fig.3 (d).

To validate the applicability of this method in the propagation characteristics of vortex beams in an inhomogeneous plasma slab, Fig.4 presents the intensity profiles of the reflected and transmitted beams from a three-layered plasma slab. In this, the incident angle is also taken as $30^{\circ}$. The radial index and topological charge are $p=0$, and $l=2$, respectively. Table1 shows the parameters. The electron number density may exist in the plasma sheath which may be formed during the flight of a high-speed spacecraft.

TABLE I

ELECTRON NUMBER DENSITY, COLLISION FREQUENCY AND THICKNESS OF THE THREE-LAYERED PLASMA SLAB

\begin{tabular}{cccc}
\hline \hline Items & $\begin{array}{c}\text { Number } \\
\text { density }\left(/ \mathrm{m}^{3}\right)\end{array}$ & $\begin{array}{c}\text { Collision } \\
\text { frequency }(\mathrm{GHz})\end{array}$ & Thickness $(\lambda)$ \\
\hline $1^{\text {st }}$ layer & $1.7 \times 10^{16}$ & 10 & 10 \\
$2^{\text {nd }}$ layer & $1.0 \times 10^{16}$ & 5 & 20 \\
$3^{\text {rd }}$ layer & $5 \times 10^{15}$ & 1 & 10 \\
\hline \hline
\end{tabular}

Fig.4 illustrates the intensity contours of the reflected and transmitted beams and it could be observed that they still keep the circular shape, whereas the distortion in the intensity of the reflected beam element is very evident. When the incident beam is TE polarized, the intensities of the TM reflected beam are smaller than that of the TE reflected beam, whereas the intensities of the TM transmitted beam are larger than that of the TE transmitted beam. However, it is different from the TM polarized incident beam.

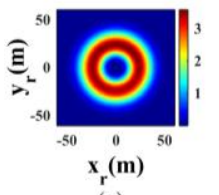

(a)

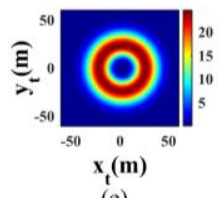

(e)

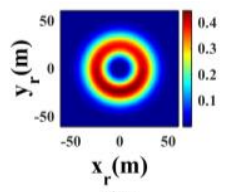

(b)

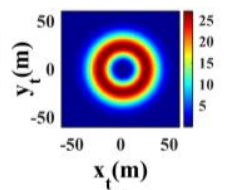

(f)

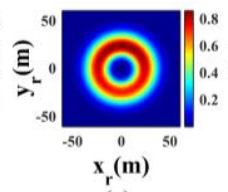

(c)

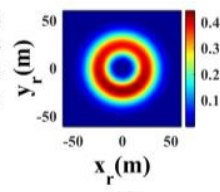

(d)
Fig.4 Intensity profiles of the reflected and transmitted beams from a three-layered plasma slab, $B_{0}=0.03 T, p=0, l=2, \theta_{i}=30^{\circ}$. The meanings of

(a)-(h) are the same as the description in Fig.3.

Based on the above observations, it is concluded from Figs. 3 and 4 that the distortion of cross-polarization components is more evident than that of principal polarization components, which may be mainly related to the magnetic flux intensity. Whereas, the contours of the transmitted electric field components have very small perturbations.

\section{B. OAM states of the reflected and transmitted beams}

To elucidate the OAM states, or spiral spectrum of the reflected and transmitted fields, their projections into the spiral harmonics $\exp (i n \phi)$ need to be computed. According to the definition of expansion of spiral spectrum [38], the electric field $U(r, \phi, z)$ can be regarded as a superposition of multiple spiral harmonics $\exp (i n \phi)$ as shown by

$$
U(r, \phi, z)=\frac{1}{\sqrt{2 \pi}} \sum_{n=-\infty}^{\infty} s_{n}(r, z) \exp (i n \phi),
$$

where $s_{n}(r, z)=1 / \sqrt{2 \pi} \int_{0}^{2 \pi} U(r, \phi, z) \exp (-i n \phi) d \phi$ and the energy of each mode is $W_{n}=\int_{0}^{\infty}\left|s_{n}(r, z)\right|^{2} r d r$. Then the weight of each OAM state is defined by the following (17):

$$
P_{n}=\frac{W_{n}}{\sum_{-\infty}^{\infty} W_{n}} .
$$

After substituting (7) and (8) into (16) and (17), the OAM weights of the reflected and transmitted field components are plotted as shown in Figs.5-8. 
In Figs.5-8, the frequency and the topological charge of the incident beam are $2 \mathrm{GHz}$ and 2, respectively. Figs.5-7 show the results of a three-layered plasma slab, where the parameters of the slab are given in Table 1.

Fig.5(a)-(d) shows that for the TE-TE reflected beam, in addition to the primary OAM state $n=-2$ which is equal to the opposite topological charge of the incident beam, the weights of two neighboring OAM states cannot be neglected, but OAM states of other reflected field components indicating very small perturbations. It is revealed in Fig.5(e)-(h) that no matter what the polarized beam is incident, although the neighboring OAM states are derived, the weights of the primary OAM state $n=2$ in the transmitted field components are all greater than 0.9 .
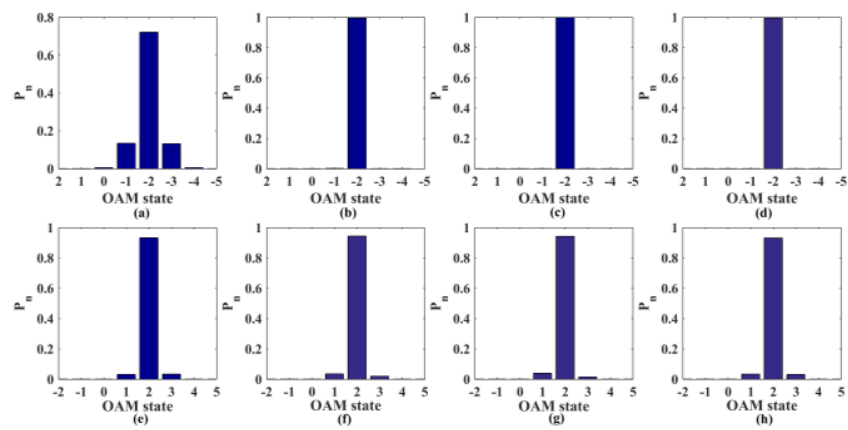

Fig.5 OAM states of the reflected and transmitted electric field components from a three-layered plasma slab. Parameters are $B_{0}=0.3 T, p=0, l=2$, and

$\theta_{i}=45^{\circ}$. The meanings of (a)-(h) are the same as the description in Fig.3.

Compared with Fig.5, the results as show in Fig.6 indicate that when the incident angle is changed from $45^{\circ}$ to $30^{\circ}$, more neighboring OAM states in the reflected field components could be noted and the weights of the primary OAM state $n=-2$ decrease, except for the TE-TE reflected beam. Otherwise, for the transmitted field components, the weights of the primary OAM state $n=2$ increase.
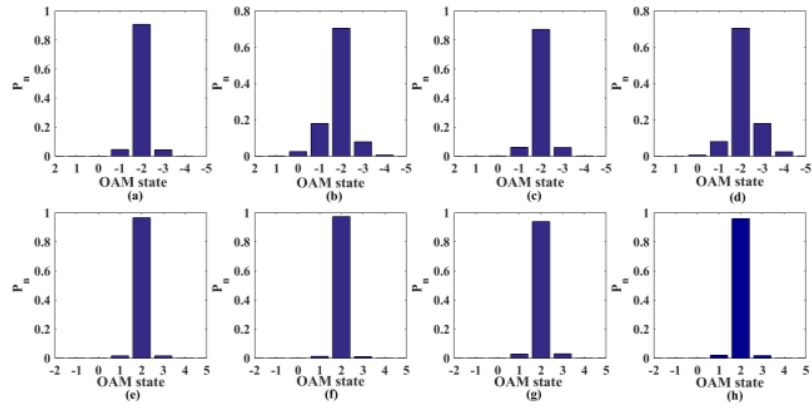

Fig.6 OAM states of the reflected and transmitted electric field components from a three-layered plasma slab. Parameters are $B_{0}=0.3 T, p=0, l=2$, and

$\theta_{i}=30^{\circ}$. The meanings of (a)-(h) are the same as the description in Fig.3.

Similarly, the parameters used in Fig.7 are the same as that of Fig.6 except for the magnetic flux intensity $B_{0}$. As the magnetic flux intensity decreases, the distortion of OAM states for both the reflected and transmitted field components is also weak. This demonstrates that the weights of the primary OAM state for the reflected beams $n=-2$ are larger.

Fig.8 shows the OAM distortion of a five-layered plasma slab, where the slab parameters are given in Table 2 .

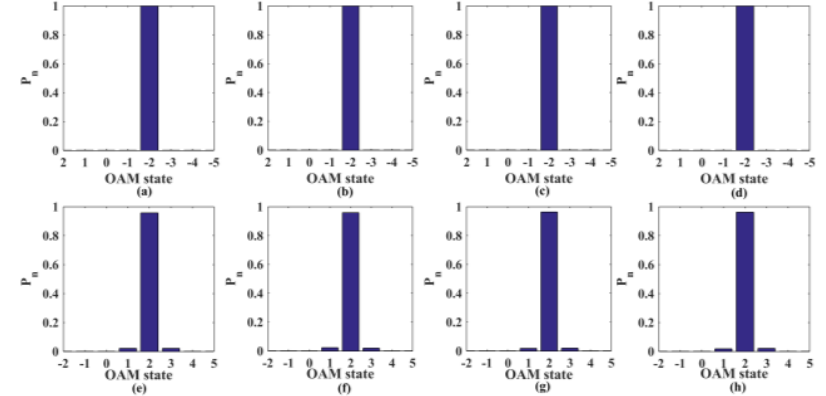

Fig.7 OAM states of the reflected and transmitted electric field components from a three-layered plasma slab. Parameters are $B_{0}=0.03 T, p=0, l=2$, and $\theta_{i}=30^{\circ}$. The meanings of (a)-(h) are the same as the description in Fig.3.

TABLE II

ELECTRON NUMBER DENSITY, COLLISION FREQUENCY AND THICKNESS OF THE FIVE-LAYERED PLASMA SLAB

\begin{tabular}{cccc}
\hline \hline Items & $\begin{array}{c}\text { Number } \\
\text { density }\left(/ \mathrm{m}^{3}\right)\end{array}$ & $\begin{array}{c}\text { Collision } \\
\text { frequency }(\mathrm{GHz})\end{array}$ & Thickness $(\lambda)$ \\
\hline $1^{\text {st }}$ layer & $1.7 \times 10^{16}$ & 10 & 10 \\
$2^{\text {nd }}$ layer & $1.0 \times 10^{16}$ & 5 & 20 \\
$3^{\text {rd }}$ layer & $5 \times 10^{15}$ & 1 & 10 \\
$4^{\text {th }}$ layer & $1.0 \times 10^{16}$ & 5 & 20 \\
$5^{\text {th }}$ layer & $1.7 \times 10^{16}$ & 10 & 10 \\
\hline \hline
\end{tabular}
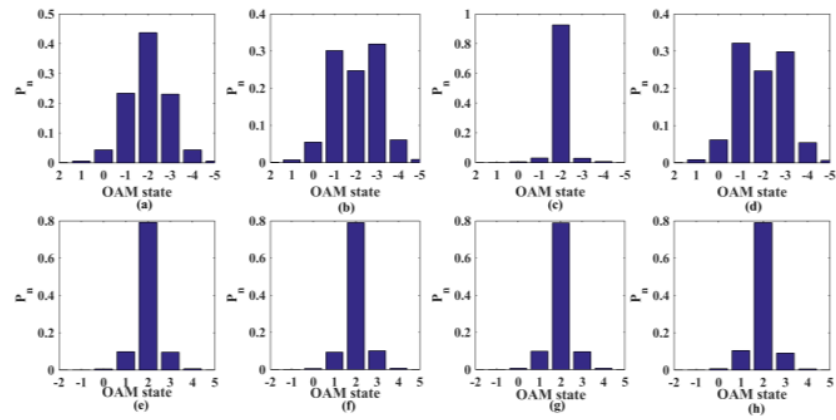

Fig.8 OAM states of the reflected and transmitted electric field components from a five-layered plasma slab. Parameters are $B_{0}=0.3 T, p=0, l=2$, and

$\theta_{i}=45^{\circ}$. The meanings of (a)-(h) are the same as the description in Fig.3.

Fig.8 illustrates that as the number of layers increases, the deformation of the OAM states of the reflected electric field components is more prominent and the weight of the primary OAM state $n=-2$ is less than 0.5 , except for the TM-TM reflected beam. It should also be noticed in this case that the weights of the primary OAM state $n=-2$ are less than those of their neighboring OAM state for the cross-polarization components as shown in Figs.8(b), (d).

For the transmitted field components, a comparison to the results of a three-layered plasma slab is shown in Figs.5. It could be observed that the weights of the primary OAM state $n=2$ decrease, while the weights of the neighboring OAM states increase.

What is noteworthy from Figs.5-8 is that although the distortion of the reflected field components is greatly influenced by the incident angle and the plasma parameters, the weights of the primary OAM state for the transmitted field 
components are larger than 0.7 , while the total weight of its neighboring OAM states is less than 0.3.

\section{Reflectance, transmittance, and absorptance of the power} from a three-layered plasma slab

The reflectance, transmittance, and absorptance of the power can be analyzed by using (9) and (10), as discussed in this section. In this, the incident beam is TE polarized. The parameters of the three-layered plasma slab as indicated in Table1 are also used here. Fig. 9 shows the variations of $R, T$, and $A$ with the incident frequencies.
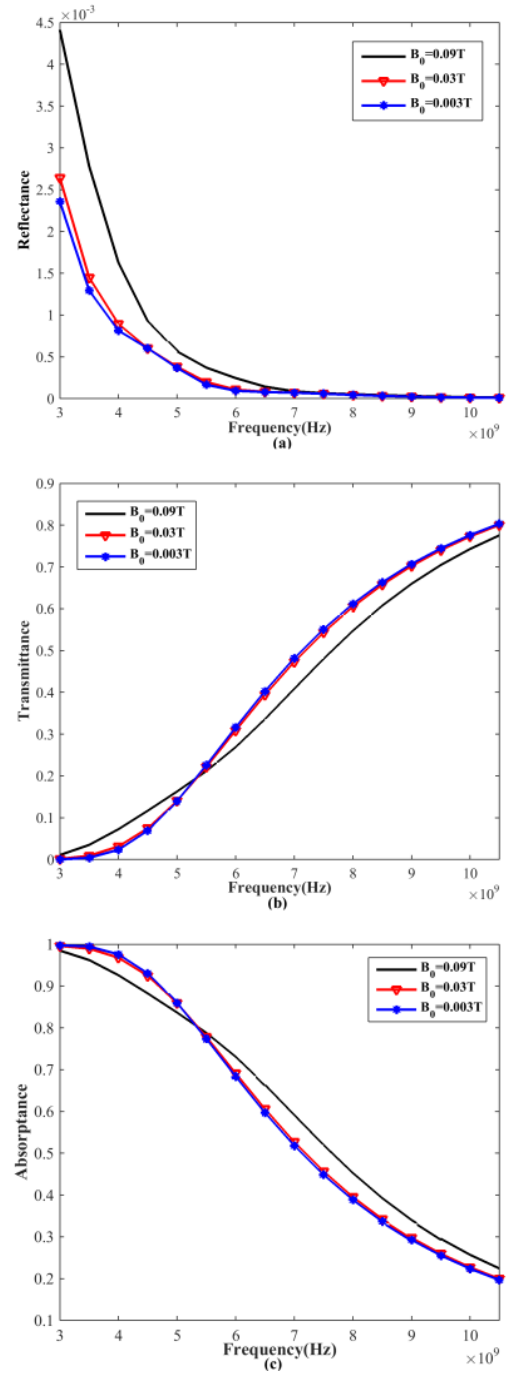

Fig.9 Variations of reflectance, transmittance, and absorptance of the power from a three-layered plasma slab with incident frequencies and $B_{0}$, (a) reflectance; (b) transmittance; (c) absorptance.

As the incident frequency is larger than the plasma frequency, the reflectance is very small. As the incident frequency increases, the reflectance and absorptance decrease, whereas the transmittance increases. For the same incident frequency, the reflectance increases with the magnetic field $B_{0}$. However, the effects of the magnetic field on transmittance depend on the frequency range of incident wave. When the incident frequency is in the range of $3-5 \mathrm{GHz}$, the transmittance increases with the magnetic field $B_{0}$, whereas for the incident wave frequency in the range of $6-10 \mathrm{GHz}$, the transmittance decreases with the magnetic field $B_{0}$.
Fig.10 shows the variations of $R, T$, and $A$ with the incident angles, where the incident frequency is $5 \mathrm{GHz}$, and the magnetic field $B_{0}$ is $0.03 \mathrm{~T}$.
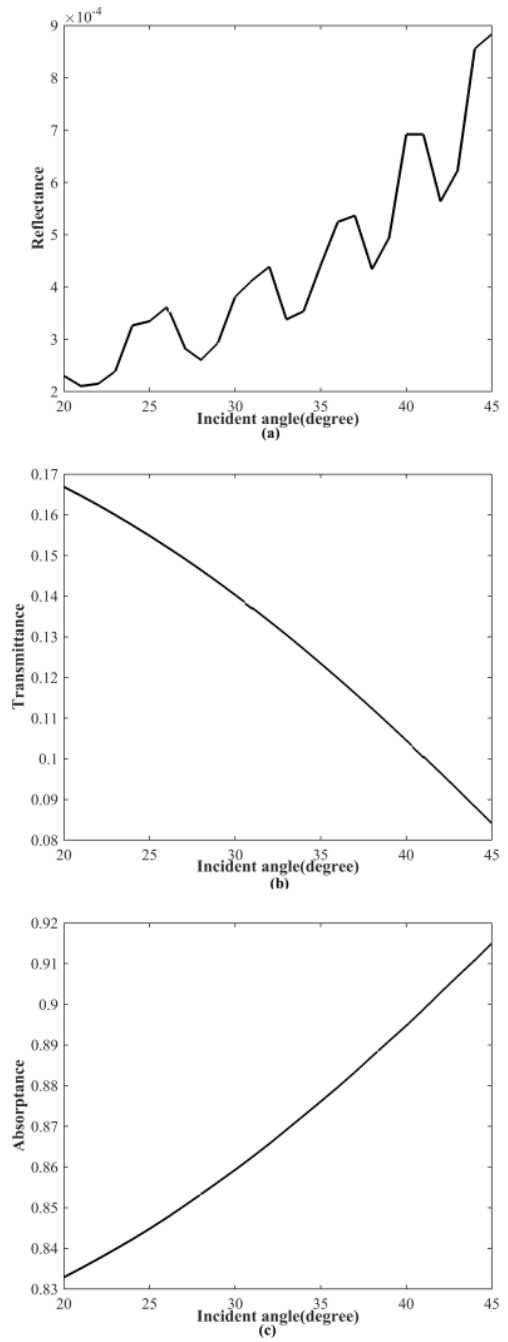

Fig.10 Variations of reflectance, transmittance, and absorptance of the power from a three-layered plasma slab with incident angles

As the incident angle increases, the reflection increases, whereas the transmission decreases. It is to be noted that both the reflectance and transmittance are small. By increasing the incident angle, the propagation distance of the wave within the plasma slab will increase, resulting in a large amount of wave energy to be absorbed by the plasma slab due to collision.

\section{CONCLUSION}

In this study, the reflection, transmission, and absorption of vortex beams in an inhomogeneous magnetized plasma slab have been investigated. The reflected and transmitted fields, the reflectance, transmittance, and absorptance of the power have been determined using both the angular spectrum expansion and the $4 \times 4$ transfer matrix methods. Based on the numerical simulation, due to the effect of magnetic field, for a linearly polarized incident beam, the reflected and transmitted beams are no longer linearly polarized and consist of principal polarization and cross-polarization components. It could be noted that the magnitudes of the cross polarization components cannot be ignored. The intensity distortion of cross polarization 
components is more evident as compared to the principal polarization components. From the results of OAM states of the reflected and transmitted beams, it could be concluded that the distortion of the reflected field components is greatly influenced by the incident angle and by the plasma parameters, whereas the primary OAM state is dominant at all time for the transmitted beam. Our numerical simulation indicated that for a TE polarized incident wave, an increase in the frequency from $3 \mathrm{GHz}$ to $10.5 \mathrm{GHz}$ results in an increase in the transmittance by a factor of 0.8 , whereas the effect on the absorptance is opposite. Therefore, it can be deduced that the transmittance can be increased by increasing the incident frequency and by decreasing the incident angle. Methods of studying the propagation of vortex beams in an inhomogeneous plasma slab provide theoretical support for the application of vortex wave communications, such as the blackout problem as observed in the flight of hypersonic vehicles.

\section{REFERENCES}

[1] L. Allen, M.W. Beijersbergen, R. J. C. Spreeuw, and J. P. Woerdman, "Orbital angular momentum of light and the transformation of LaguerreGaussian laser modes," Phys. Rev. A, vol. 45, no. 11, pp. 8185-8189, June, 1992.

[2] H. Okuda and H. Sasada, "Significant deformations and propagation variations of Laguerre-Gaussian beams reflected and transmitted at a dielectric interface," J. Opt. Soc. Am. A, vol. 25, no. 4, pp. 881-890, April, 2008.

[3] E. Karimi, S. A. Schulz, I. De Leon, H. Qassim, J. Upham, and R. W. Boyd, "Generating optical orbital angular momentum at visible wavelengths using a plasmonic metasurface," Light Sci. Appl., vol. 3, no.5, pp. e167, May, 2014.

[4] W. B. Wang, R. Gozali, L. Shi, L. Lindwasser, and R. R. Alfano, "Deep transmission of Laguerre-Gaussian vortex beams through turbid scattering media," Opt. Lett., vol. 41, no. 9, pp. 2069-2072, May, 2016.

[5] G. Foo, D. M. Palacios, Jr.G.A. Swartzlander, "Optical vortex corona graph," Opt. Lett., vol. 30, no. 24, pp. 3308-3310, Dec., 2005.

[6] N. s. Uribe-Patarroyo, A. Fraine, D. S. Simon, O. Minaeva, and A. V. Sergienko, "Object Identification Using Correlated Orbital Angular Momentum States," Phys. Rev. Lett., vol. 110, no.4, pp.043601, Jan., 2013.

[7] B. Thidé, H. Then, J. Sjöholm, K. Palmer, J. Bergman, T. D. Carozzi, Ya. N. Istomin, N. H. Ibragimov, and R. Khamitova, "Utilization of Photon Orbital Angular Momentum in the Low-Frequency Radio Domain," Phys. Rev. Lett., vol. 99, no. 8, pp.087701, August, 2007.

[8] F. Tamburini, E. Mari, B. Thidé, C. Barbieri, and F. Romanato, "Experimental verification of photon angular momentum and vorticity with radio techniques," Appl. Phys. Lett., vol. 99, pp. 204102, 2011.

[9] P. Schemmel, G. Pisano, and B. Maffei, "Modular spiral phase plate design for orbital angular momentum generation at millimeter wavelengths," Opt. Express, vol.22, no.12, pp.14712, June, 2014.

[10] L. Cheng, W. Hong, Z. Hai, "Generation of Electromagnetic Waves with Arbitrary Orbital Angular Momentum Modes," Sci. Rep., vol. 4, pp. 4814, April, 2014.

[11] Y. Pan, S. Zheng, J. Zheng, Y. Li, X. Jin, H. Chi, and X. Zhang, "Generation of Orbital Angular Momentum Radio Waves Based on Dielectric Resonator Antenna," IEEE Antennas Wireless Propag. Lett. , vol. 16, pp.385-388, 2017.

[12] Y. Yan, G. Xie, M. P. J. Lavery, H. Huang, N. Ahmed, C. Bao, et al, "High-capacity millimeter-wave communications with orbital angular momentum multiplexing," Nat. Commun., vol. 5, pp. 4876, Sept., 2014.

[13] F. E. Mahmouli, S. D. Walker, "4-Gbps Uncompressed Video Transmission over a $60-\mathrm{GHz}$ Orbital Angular Momentum Wireless Channel," IEEE Wireless Commun. Lett. , vol.2, no.2, pp. 223-226, April, 2013.

[14] X. Hui, S. Zheng, Y. Chen, Y. Hu, X. Jin, H. Chi, et al. "Multiplexed Millimeter Wave Communication with Dual Orbital Angular Momentum (OAM) Mode Antennas," Sci. Rep. , vol. 5, pp.10148, May, 2015.

[15] F. Spinello, C. G. Someda, R. A. Ravanelli, E. Mari, G. Parisi, F. Tamburini, et al., "Radio channel multiplexing with superpositions of opposite-sign OAM modes," AEU-INT J ELECTRON. C., vol. 70, no. 8, pp. 990-997, August, 2016.

[16] K. Liu, Y. Cheng, Z. Yang, H. Wang, Y. Qin, and X. Li, "Orbital-Angular-Momentum-Based Electromagnetic Vortex Imaging," IEEE Antennas Wireless Propag. Lett., vol. 14, pp. 711-716, 2015.

[17] T. Yuan, Y. Cheng, H. Wang, and Y. Qin, "Electromagnetic Vortex-Based Radar Imaging Using a Single Receiving Antenna: Theory and Experimental Results," Sensors, p.17630, 2017.

[18] S. J. Briczinski, P. A. Bernhardt, C. L. Siefring, S.-M. Han, T. R. Pedersen, and W. A. Scales, ““'Twisted Beam” SEE Observations of Ionospheric Heating from HAARP," Earth Moon Planets, vol. 116, no. 1, pp. 55-66, Oct., 2015.

[19] T. B. Leyser, F. Waldemarsson, S. C. Buchert, "Plasma angular momentum effects and twisted incoherent scatter radar beams," Radio Sci., vol. 47, no. 5, pp. RS5004, Oct., 2012.

[20] T. B. Leyser, L. Norin, M. McCarrick, T. R. Pedersen, and B. Gustavsson, "Radio Pumping of Ionospheric Plasma with Orbital Angular Momentum," Phys. Rev. Lett., vol. 102, no.6, pp. 065004, Feb., 2009.

[21] V. L. Ginzberg, "The propagation of Electromagnetic waves in Plasma," Pergammon, New York, 1970.

[22] D. L. Tang, A. P. Sun, X. M. Qiu, and P. K. Chu, "Interaction of Electromagnetic Waves With a Magnetized Nonuniform Plasma Slab," IEEE Trans. Plasma Sci., vol. 31, no.3, pp. 405-410, June, 2003.

[23] M. Wang, H. Li, Y. Dong, G. Li, B. Jiang, Q. Zhao, et al., "Propagation Matrix Method Study on THz Waves Propagation in a Dusty Plasma Sheath," IEEE Trans. Antennas Propag. , vol. 64, no.1, pp. 286-290, Jan., 2016.

[24] B. Jazi, Z. Rahmani, and B. Shokri, "Reflection and Absorption of Electromagnetic Wave Propagation in an Inhomogeneous Dissipative Magnetized Plasma Slab," IEEE Trans. Plasma Sci., vol. 41, no.2, pp. 290-295, Feb., 2013.

[25] A. Samimi and J. J. Simpson, "An Efficient 3-D FDTD Model of Electromagnetic Wave Propagation in Magnetized Plasma," IEEE Trans. Antennas Propag., vol. 63, no. 1, pp. 269-279, Jan., 2015.

[26] J. H. Lee and D. K. Kalluri, "Three-Dimensional FDTD Simulation of Electromagnetic Wave Transformation in a Dynamic Inhomogeneous Magnetized Plasma," IEEE Trans. Antennas Propag., vol. 47, no.7, pp. 1146-1151, July, 1999.

[27] P. D. Cannon and F. Honary, "A GPU-Accelerated Finite-Difference Time-Domain Scheme for Electromagnetic Wave Interaction with Plasma," IEEE Trans. Antennas Propag. , vol. 63, no. 7, pp. 3042-3054, July, 2015.

[28] J.-F. Liu, X.-L. Xi, G.-B. Wan, and L.-L. Wang, "Simulation of Electromagnetic Wave Propagation Through Plasma Sheath Using the Moving-Window Finite-Difference Time-Domain Method " IEEE Trans. Plasma Sci., vol. 39, no. 3, pp. 852-855, Mar., 2011.

[29] O. Sakai and K. Tachibana, "Properties of Electromagnetic Wave Propagation Emerging in 2-D Periodic Plasma Structures," IEEE Trans. Plasma Sci., vol. 35, no. 5, pp. 1267-1273, Oct., 2007.

[30] Y. Bo, Q. Zhao, X. G. Luo, Y. Liu, Y.-X. Chen, J.-W. Liu, "Study on transmission characteristics of electromagnetic waves in inhomogeneously magnetized plasma sheath," Acta Phys. Sin-CH. ED, vol. 65 , no. 3, pp. 035201 , Mar., 2016

[31] D. W. Berreman, "Optics in Stratified and Anisotropic Media: $4 * 4$ Matrix Formulation," J. Opt. Soc. Am., vol. 62, no.4, pp.502-510, April, 1972.

[32] P. Yeh, "Electromagnetic propagation in birefringent layered media," $J$. Opt. Soc. Am., vol. 69, no. 5, pp. 742-756, May, 1979.

[33] J. Ou, Y. Jiang, J. Zhang, and Y. He, "Reflection of Laguerre-Gaussian beams carrying orbital angular momentum: a full Taylor expanded solution," J. Opt. Soc. Am. A, vol. 20, 2561-2570, Dec., 2013.

[34] M. Lax, W. H. Louisell, and W. B. McKnight, "From Maxwell to paraxial wave optics," Phys. Rev. A, vol.11, pp.1365-1370, April, 1975.

[35] H. Wohler, G. Haas, M. Fritsch, and D. A. Mlynski, "Faster $4 * 4$ matrix method for uniaxial inhomogeneous media," J. Opt. Soc. Am. A, vol. 5, no.9, pp.1554-1557, Sep., 1988.

[36] G. D. Landry and T. A. Maldonado, "Gaussian beam transmission and reflection from a general anisotropic multilayer structure," Appl. Optics, vol.35, no.30, pp.5870-5879, Oct., 1996.

[37] F. Wang, D. Ge, and B. Wei, "SO-FDTD method for computation of reflection and transmission coefficients for magnetized plasma layer," Chin. J. Radio, vol.23, no.4, pp.704-707, Aug., 2008.

[38] Y Jiang, S Wang, J Zhang, J Ou, H Tang. "Spiral spectrum of LaguerreGaussian beam propagation in non-Kolmogorov turbulence," Opt. Commun., vol.303, no.16, pp. 38-41, Aug., 2013 